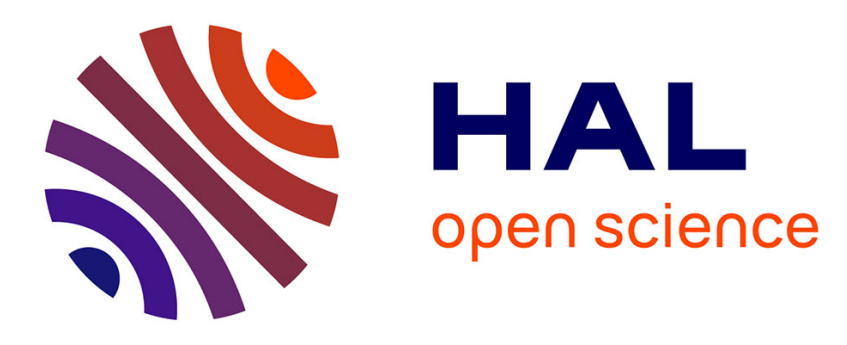

\title{
Residence times and boundary-following behavior in animals
}

Sebastian Weitz, Stéphane Blanco, Richard A Fournier, Jacques Gautrais, Christian Jost, Guy Theraulaz

\section{- To cite this version:}

Sebastian Weitz, Stéphane Blanco, Richard A Fournier, Jacques Gautrais, Christian Jost, et al.. Residence times and boundary-following behavior in animals. Physical Review E: Statistical, Nonlinear, and Soft Matter Physics, 2014, 89 (5), 10.1103/PhysRevE.89.052715 . hal-02324606

\section{HAL Id: hal-02324606 \\ https://hal.science/hal-02324606}

Submitted on 22 Oct 2019

HAL is a multi-disciplinary open access archive for the deposit and dissemination of scientific research documents, whether they are published or not. The documents may come from teaching and research institutions in France or abroad, or from public or private research centers.
L'archive ouverte pluridisciplinaire HAL, est destinée au dépôt et à la diffusion de documents scientifiques de niveau recherche, publiés ou non, émanant des établissements d'enseignement et de recherche français ou étrangers, des laboratoires publics ou privés. 


\title{
Residence times and boundary-following behavior in animals
}

\author{
Sebastian Weitz, ${ }^{1,2,3}$ Stéphane Blanco, ${ }^{1,2}$ Richard Fournier, ${ }^{1,2}$ Jacques Gautrais, ${ }^{4,5}$ Christian Jost, ${ }^{4,5}$ and Guy Theraulaz ${ }^{4,5}$ \\ ${ }^{1}$ Laboratoire Plasma et Conversion d'Energie, UMR-CNRS 5213, Université Paul Sabatier, Bâtiment 3R1, 118 Route de Narbonne, \\ F-31062 Toulouse cedex 9, France \\ ${ }^{2}$ Laboratoire Plasma et Conversion d'Energie, CNRS, F-31062 Toulouse cedex 9, France \\ ${ }^{3}$ Zentrum für Informationsdienste und Hochleistungsrechnen, Technische Universität Dresden, Zellescher Weg 12, 01069 Dresden, Germany \\ ${ }^{4}$ Centre de Recherches sur la Cognition Animale, UMR-CNRS 5169, Université Paul Sabatier, Bâtiment 4R3, \\ 118 Route de Narbonne, F-31062 Toulouse cedex 9, France \\ ${ }^{5}$ CNRS, Centre de Recherches sur la Cognition Animale, F-31062 Toulouse cedex 9, France \\ (Received 19 November 2013; revised manuscript received 9 April 2014; published 30 May 2014)
}

\begin{abstract}
Many animals in heterogeneous environments bias their trajectories displaying a preference for the vicinity of boundaries. Here we propose a criterion, relying on recent invariance properties of residence times for microreversible Boltzmann's walks, that permits detecting and quantifying boundary-following behaviors. On this basis we introduce a boundary-following model that is a nonmicroreversible Boltzmann's walk and that can represent all kinds of boundary-following distributions. This allows us to perform a theoretical analysis of field-resolved boundary following in animals. Two consequences are pointed out and are illustrated: A systematic procedure can now be used for extraction of individual properties from experimental field measurements, and boundary-curvature influence can be recovered as an emerging property without the need for individuals perceiving the curvature via complex physiological mechanisms. The presented results apply to any memoryless correlated random walk, such as the run-and-tumble models that are widely used in cell motility studies.
\end{abstract}

DOI: 10.1103/PhysRevE.89.052715 PACS number(s): 87.19.rs, 05.40.Fb, 87.10.Mn, 05.60.Cd

\section{INTRODUCTION}

Animal movement has inspired numerous theoretical and empirical papers in ecology over the past decades [1,2]. At all scales, and most of the time, moving animals interact with the local environment, albeit with stochasticity [3]. The ability of organisms to orientate within heterogeneous and patchy landscapes is highly relevant for exploration and foraging strategies [4-6]. Typical examples are when animals display oriented movement patterns at multiple scales [7], switch among behavioral modes in response to different environmental conditions [8], or tend to restrain their paths within a home range [9]. In this paper we address the case in which animals react specifically to physical or patch boundaries, in particular boundary-following behaviors [10-17], that are commonly referred to as thigmotactism in the behavioral biology literature $[18,19]$. The relation between individual trajectories and broad-scale population distributions is still an open question for such boundary-following animals. Understanding the residence times as well as the spatial and angular distributions of animals moving in the vicinity of the boundary is however a prerequisite to investigate many processes occurring within the near-boundary zone. For instance, these statistics determine the information that can be sampled by an individual animal (in particular when it has only local perception of its environment) or condition the probability of encountering a congener. Therefore they play an important role in foraging, settling, and information sharing among conspecifics [6,20-22]. Finally they occur over a large spectrum of spatial scales from the places where social insects can drop building material when establishing a new nest [23] to the long-distance dispersal of seeds by birds [24] and marine landscape ecology [25-27]. Experimental data about the spatial and directional distributions of animals moving in the vicinity of boundaries have been scarce in the past but now become more and more available [7,28-31] and make urgent the need for related modeling works.

Models of boundary-following behaviors in animals usually assume a behavioral switch between free-field movements (within a two- or three-dimensional domain) and movements along the boundary (which is one dimension smaller). Exact solutions for the resulting stationary walker repartition between the boundary and the free field have been obtained, e.g., in Refs. [10,11]. However, in these models, when organisms follow the boundary, they are not merely influenced by the boundary: They are modeled as located on the boundary itself. These models (also called wall-following models) are therefore inadequate to analyze the now available spatial and angular statistical observations, such as those of Fig. 1. This leads us to propose a modeling alternative for boundary-induced behaviors where boundary followers move in a domain of same dimension as when in free-field mode with only their direction-change decisions influenced by their perception of the boundary. This also means that the effect of the boundary is no more considered to be strictly local but extends to some distance from the boundary. In addition to the already mentioned benefits of such fully spatialized models of boundary following (in particular, the possibility to infer individual behaviors from spatially resolved experimental data), they allow for understanding different residence-time statistics as emerging under the constraint of different boundary geometries with behaviors otherwise unchanged: It is not required that the behavioral model depends on the perception of advanced geometrical features, such as boundary curvature. Fully spatialized models are scarce in the context of boundary-following modeling. The few related papers are restricted to very specific situations (clusters of interacting self-propelled rods gliding along a wall [32] or constantly turning particles with a nonvanishing mean torque 
(a)

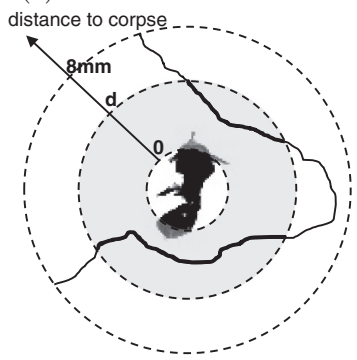

(c)

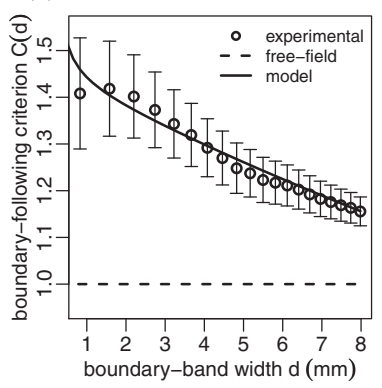

(b)

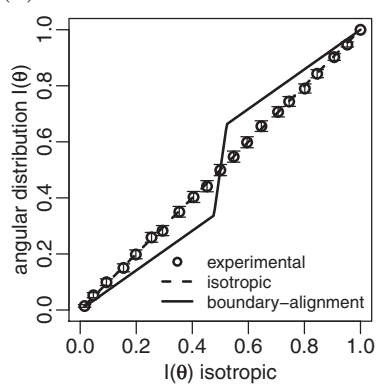

(d)

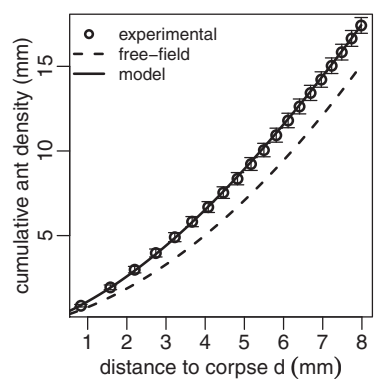

FIG. 1. Modeling boundary following in an experimental study of ants Messor sancta forming cemeteries [59]. (a) Trajectory. Typical trajectory of an ant in the vicinity of a conspecific's corpse. (b) Angular distribution. Considering criterion 1 (defined in the main text) we have calculated the normalized cumulative $I(\theta)$ of $\tilde{f}_{\text {stat }}(d, \theta)|\cos (\theta)|$ for $\theta \in[0, \pi]$ where we used the circular symmetry around the corpse to write the stationary distribution function $f_{\text {stat }}(\boldsymbol{r}, \boldsymbol{\omega}) \equiv \tilde{f}_{\text {stat }}(d, \theta)$ with $d$ being the distance to the corpse (approximated by a disk of radius $2 \mathrm{~mm}$ ) and $\theta$ being the absolute angle between $\boldsymbol{\omega}$ and radial vector $\boldsymbol{e}_{\boldsymbol{r}}$. The plot shows $I(\theta)$ as a function of $I(\theta)$ calculated for an isotropic distribution. In the case of isotropy (dashed curve), this corresponds to a straight line of unit slope, whereas in the case of boundary alignment [solid curve, Eq. (2) with $\phi(\boldsymbol{r}, \boldsymbol{\omega})=10$ if $\left\|\boldsymbol{\omega} \cdot \boldsymbol{e}_{\boldsymbol{r}}\right\|<0.3$ and $\phi(\boldsymbol{r}, \boldsymbol{\omega})=1$ else, note that $\boldsymbol{\alpha}(\boldsymbol{r})$ and $K(\boldsymbol{r})>0$ do not impact $I(\theta)$ ], the curve deviates from the straight line. The experimental ant distribution (circles, mean \pm SEM for $n=625$ trajectories, distance $d=5 \mathrm{~mm}$ ) is isotropic. This conclusion has also been confirmed for other distances $d$ (from 0 to $8 \mathrm{~mm}$ ). (c) Residence time. Considering criterion 2, we have calculated the boundary-following criterion $\mathcal{C}=\frac{\langle T\rangle}{\langle T\rangle_{\text {ref }}}$ (defined in the main text). An ant is tracked as long as its distance to the corpse is shorter than $8 \mathrm{~mm}$, and the boundary-following time $T$ is defined as the time spent within the band of width $d$ around the corpse during this track [shaded zone and thick parts of trajectory in (a)]. The presented experimental curve $\mathcal{C}(d)$ (circles, mean \pm SEM, 507 trajectories) indicates boundary-following behavior $(\mathcal{C} \in[1.2,1.4]$ compared to 1 expected for free-field behavior) and can be fitted with our model for $\frac{\hat{\alpha}}{1-g}=46 e_{r} \mathrm{~m}^{-1}$ [solid curve, see Eq. (2)]. (d) Spatial distribution. Alternatively, criterion 2 can be considered (without any path tracking) by looking at the ant density profile around a corpse. Here $\hat{\eta}(u)$ is the surfacic ant density at the stationary state, averaged over the contour corresponding to the distance $u$ to the corpse. The figure shows the cumulative ant density $\frac{c}{\Phi_{\delta \Omega, \text { stat }}} \int_{0}^{d} \hat{\eta}(u) 2 \pi u d u$, normalized by the incoming ant flux $\hat{\phi}_{\delta \Omega \text {,stat }}$ at $d=8 \mathrm{~mm}$ and by the mean ant speed $c$. Thus $\hat{\eta}(u)$ is proportional to the derivatives of the curves. The observed ant density (circles, mean \pm SEM) is about $30 \%$ higher than expected for free-field behavior (dashed curve), thus demonstrating boundary following and is again in close agreement with our model fit (solid curve).

[33]), and the spatial and angular distributions of the particles resulting from these models are not analytically tractable. Some recent papers in statistical physics have considered the motion of self-propelled particles in the presence of many randomly distributed obstacles [34-37]. However, these papers have focused on larger scales and have only marginally investigated the distributions of the particles in the vicinity of the boundary of an obstacle. Boundary following has also been investigated in robotics, but the models considered in this field are generally deterministic and are therefore less adequate for describing animal behavior (see Ref. [38] and references therein). Therefore, rather than by the boundary-following literature, our approach is inspired by models used for studying bacteria, cells, or animals moving up a stimulus gradient (e.g., a chemical gradient for Escherichia coli bacteria [39], the slime mold Dictyostelium discoideum and leukocytes [40,41], an electrical gradient for kertinocytes involved in wound healing [42], or a prey gradient in prey-predator models for some protozoa [43]).

The first delicate question in this context is the criterion to be used for the experimental detection and quantification of boundary-following behaviors in contrast with unoriented movements or only boundary-reflection behavior. We here propose a criterion inspired by recently established invariance properties of microreversible Boltzmann's walks (which have been identified in many biological contexts where they are also referred to as run-and-tumble walks, Pearson's walks, or discrete correlated random walks with exponentially distributed free paths [44-47]). Then, on the basis of this criterion, we introduce a boundary-following model that is a nonmicroreversible Boltzmann's walk and that can represent all kinds of boundary-following behaviors. Finally, we formulate two inversion properties permitting to infer individual behaviors from experimentally observed spatial and angular distributions.

We start by restricting the scope to organisms that can be modeled as constant-speed Boltzmann's walkers, meaning that their distribution function satisfies the following kinetic equation:

$$
\frac{\partial f}{\partial t}+c \boldsymbol{\omega} \cdot \boldsymbol{\nabla} f=-v f+\int v^{\prime} f^{\prime} p\left(\boldsymbol{\omega} \mid \boldsymbol{\omega}^{\prime}\right) d \boldsymbol{\omega}^{\prime},
$$

where $f \equiv f(\boldsymbol{r}, \boldsymbol{\omega}, t)$ is the distribution function of location $\boldsymbol{r}$ and movement direction $\omega$ at time $t, c$ is the constant movement speed, $v \equiv v(\boldsymbol{r}, \boldsymbol{\omega})$ is the direction-change frequency, and $p\left(\boldsymbol{\omega} \mid \boldsymbol{\omega}^{\prime}\right) \equiv p\left(\boldsymbol{\omega} \mid \boldsymbol{\omega}^{\prime}, \boldsymbol{r}\right)$ is the direction-change phase function, i.e., the probability density that when changing direction an organism picks up the new direction $\omega$ given the incoming direction $\omega^{\prime}$. The two terms on the right-hand side of this equation are so-called "collision" terms describing the effect of organisms changing their direction on the population of organisms moving in direction $\omega$ at location $r$ : The first term is the extinction term corresponding to organisms departing from this population, and the second integral term is the source term corresponding to organisms entering this population coming from all other directions $\omega^{\prime}$. The notations $f^{\prime} \equiv f\left(\boldsymbol{r}, \boldsymbol{\omega}^{\prime}, t\right)$ and $v^{\prime} \equiv v\left(\boldsymbol{r}, \boldsymbol{\omega}^{\prime}\right)$ are used to simplify this integral source term. The biological meaning of such walks is that organisms move at constant speed along straight lines 
until they perform an instantaneous direction change. Free paths (the distances between successive direction changes) are exponentially distributed, which is formalized in Eq. (1) via the use of local direction-change frequencies. This means that organisms behave statistically only as a function of their instantaneous state, location, and direction (they make no use of the memory they may have of their movement history).

\section{BOUNDARY-FOLLOWING CRITERION}

As far as the boundary-following criterion is concerned, two definitions look straightforward: organisms follow the boundary if (criterion 1) in the vicinity of the boundary, they orient their movements parallelly to the boundary (or close to the parallel), or if (criterion 2) although walking at similar speeds, they spend more time in the vicinity of the boundary than they would in a region of the same extension but located far from the boundary (in the free field). Criterion 1 best refers to processes in which animals or cells adapt their heading in reaction to a directional information, e.g., the tangent to the limit of a geometrical domain. If such a process is likely to make an animal also fulfill criterion 2, the reverse is however not true. For example, Fig. 1 describes the results of an experimental study with ants forming cemeteries, the boundary considered here being the contour of a dead ant. According to the first criterion, these ants do not display boundary-following behavior since their distribution function is quasi-isotropic, even close to the dead ant. However, their average residence time $\langle T\rangle$ within an $8 \mathrm{~mm}$ band along the boundary (defined as the time until first return to the limit of the near-boundary region) is significantly greater than it would be within the same band in free field while their movement speed remains quasi-identical. Hence, they do display boundary-following behavior according to the second criterion. We will see that this apparent contradiction can be solved with the physical picture of a stationary state combining a stratified density field with an isotropic velocity distribution (just as in a stratified gas at equilibrium with external forces).

Because it is more general and because we want to inquire into the determinants of residence times, we retain the second criterion. As illustrated in Fig. 1, the strength of boundary-following behaviors will be measured by how much the ratio $\mathcal{C}=\frac{\langle T\rangle}{\langle T\rangle_{\text {ref }}}$ exceeds unity with $\langle T\rangle_{\text {ref }}=\frac{1}{c} \frac{\pi S}{P}$ in the two-dimensional case (where $S$ is the surface of the near-boundary region and $P$ is the length of its limit) and $\langle T\rangle_{\text {ref }}=\frac{1}{c} \frac{4 V}{S}$ in the three-dimensional case (where $V$ is the volume of the near-boundary region and $S$ is the surface of its limit). This choice is motivated by invariance properties of Boltzmann's random walks: In the reference case where the walkers have no orientation information (i.e., when they display free-field behaviors) and where the boundary conditions at the limit of $\mathcal{D}$ are compatible with a locally isotropic stationary state, an isotropic homogeneous equilibrium is reached and $\langle T\rangle=\langle T\rangle_{\text {ref }}[48,49]$. Note that this invariance property and therefore the scope of our boundary-following criterion also applies to all other microreversible random walks leading to an isotropic homogeneous equilibrium (the proof, inspired by Ref. [50], is given in the Appendix). The reason why the assumption of unoriented biological walkers leads to an isotropic homogeneous equilibrium is that such walkers are assumed to display a behavioral symmetry with the direction-change frequency $v$ independent of $\boldsymbol{\omega}$ [we note $\left.v(\boldsymbol{r}, \boldsymbol{\omega})=v_{\infty}(\boldsymbol{r})\right]$ and the phase function $p$ depending on $\boldsymbol{\omega}$ only via any pair function of the deviation angle [we note $\left.p\left(\boldsymbol{\omega} \mid \boldsymbol{\omega}^{\prime}, \boldsymbol{r}\right)=p_{\infty}\left(\boldsymbol{\omega} \cdot \boldsymbol{\omega}^{\prime}, \boldsymbol{r}\right)\right]$. This implies that the directionchange statistics are microreversible [i.e., $v(\boldsymbol{r}, \boldsymbol{\omega}) p\left(\boldsymbol{\omega}^{\prime} \mid \boldsymbol{\omega}, \boldsymbol{r}\right)=$ $\left.v\left(\boldsymbol{r},-\boldsymbol{\omega}^{\prime}\right) p\left(-\boldsymbol{\omega} \mid-\boldsymbol{\omega}^{\prime}, \boldsymbol{r}\right)\right]$, and this microreversibility leads to a uniform and isotropic stationary distribution $f_{\text {stat }}$ (which is a consequence of Boltzmann's $H$ theorem). Therefore, the wide class of Boltzmann's walks in which the direction-change statistics are modulated by the boundary or vary in space, but still remain microreversible, cannot lead to boundary following in the sense of our criterion because the average residence times remain the same as in the free field $(\mathcal{C}=1)$.

\section{BOUNDARY-FOLLOWING MODEL}

These invariance properties of microreversible memoryless Boltzmann's walks show that increasing $\langle T\rangle_{\Omega \text {,stat }}$ cannot be achieved by modifying the direction-change frequency $v$ or the phase function $p$ without breaking microreversibility. Modeling boundary-following behaviors (preserving the constantspeed, instantaneity, and no memory-usage assumptions) requires therefore to work on how $v$ and $p$ are influenced by some directional perception of the boundary in such a way that $v(\boldsymbol{r}, \boldsymbol{\omega}) p\left(\boldsymbol{\omega}^{\prime} \mid \boldsymbol{\omega}, \boldsymbol{r}\right) \neq v\left(\boldsymbol{r},-\boldsymbol{\omega}^{\prime}\right) p\left(-\boldsymbol{\omega} \mid-\boldsymbol{\omega}^{\prime}, \boldsymbol{r}\right)$. All kinds of combined $v$ and $p$ dependencies can be explored. We here propose a model in which only the direction-change frequency is influenced. We therefore assume that $p=p_{\infty}$, where $p_{\infty}$ is measured under free-field conditions. The remaining question is then how $v$ depends on location and direction. This dependency needs to break microreversibility [which here means that $v(\boldsymbol{r},-\boldsymbol{\omega}) \neq v(\boldsymbol{r}, \boldsymbol{\omega})$ because $p_{\infty}$ depends only on $\boldsymbol{\omega} \cdot \boldsymbol{\omega}^{\prime}$ ], leading to a nonuniform potentially anisotropic stationary distribution of walkers. We propose the following formulation of the direction-change frequency:

$$
v(\boldsymbol{r}, \boldsymbol{\omega})=\frac{K(\boldsymbol{r})}{\phi(\boldsymbol{r}, \boldsymbol{\omega})}-\frac{c}{1-g} \boldsymbol{\alpha}(\boldsymbol{r}) \cdot \boldsymbol{\omega},
$$

where $g=\int_{\mathcal{S}} \boldsymbol{\omega} \cdot \boldsymbol{\omega}^{\prime} p_{\infty}\left(\boldsymbol{\omega} \cdot \boldsymbol{\omega}^{\prime}\right) d \boldsymbol{\omega}$ is the asymmetry parameter of the phase function (the average value of the deviationangle cosine, independent of $\omega^{\prime}$ by definition of $\left.p_{\infty}\right)$. $\phi$ and $\boldsymbol{\alpha}$ are adjustable functions with the only constraints that $\int_{\mathcal{S}} \phi(\boldsymbol{r}, \boldsymbol{\omega}) d \boldsymbol{\omega}=1, \int_{\mathcal{S}} \phi(\boldsymbol{r}, \boldsymbol{\omega}) \boldsymbol{\omega} d \boldsymbol{\omega}=\mathbf{0}(\phi$ is a normalized null-flux function of $\boldsymbol{\omega}$ ), and $v$ remains positive for all $\boldsymbol{r}$ and all $\omega . K(\boldsymbol{r})$ is a free scalar field (with the only constraint that $v$ remains positive) that does not impact the stationary distribution of the walkers and therefore does not modify the average residence times (see the Appendix). This direction-change frequency model permits obtaining all kinds of stationary anisotropic nonuniform distributions of the walkers and all kinds of residence times (see Fig. 1).

\section{INVERSION PROPERTIES}

Reciprocally, when interpreting boundary-following experiments, measuring the stationary distribution of walkers may allow for inferring the form of the $v$ dependency (assuming again that only the direction-change frequency $v$, but not the phase function $p$, is influenced by the boundary). Inversion 
would need to be performed numerically in most cases to derive the $\phi$ and $\boldsymbol{\alpha}$ dependencies from the observed stationary distribution $f_{\text {stat }}$, but we can formulate theoretical inversion properties in two cases that can be used in many of the situations encountered in experiments.

The first inversion property concerns the case when the angular distribution of the walkers at stationary state $\hat{\phi}=\frac{f_{\text {stat }}}{\eta}$ (with $\eta=\int_{\mathcal{S}} f_{\text {stat }} d \omega$ ) is observed to be independent of space in a given geometrical domain and when this domain is sufficiently extended (small Knudsen limit $\mathrm{Kn} \ll 1$ ) so that the walkers can be assumed to behave as their transport equivalents $\left[p_{\infty}\right.$ replaced by an isotropic phase function and $v$ replaced by $v^{*}=v(1-g)$ in the Boltzmann walk]. The small Knudsen limit is not required if the phase function $p_{\infty}$ is isotropic. In this case, measuring $\hat{\phi}$ and $\hat{\boldsymbol{\alpha}}=\frac{\nabla \eta}{\eta}$ (the relative value of the observed density gradient) allows for stating that walkers change direction according to $v$ following Eq. (2) with $\phi=\hat{\phi}$ and $\boldsymbol{\alpha}=\hat{\boldsymbol{\alpha}}$, only the $K$ scalar field remaining adjustable (meaning that the inversion solution is not unique). A possible proof consists of first checking that the stationary version of Eq. (1) with $p\left(\boldsymbol{\omega} \mid \boldsymbol{\omega}^{\prime}\right)$ isotropic is indeed satisfied when replacing $v$ by the expression of Eq. (2). Then, assuming that another direction-change frequency $\tilde{v}$ is also compatible with both Eq. (1) and the observed stationary distribution, writing $\tilde{v}=v+\zeta$ and replacing $v$ by $v+\zeta$ in Eq. (1) leads to

$$
\zeta(\boldsymbol{r}, \boldsymbol{\omega}) \hat{\phi}(\boldsymbol{\omega})=\int_{\mathcal{S}} \zeta\left(\boldsymbol{r}, \boldsymbol{\omega}^{\prime}\right) \hat{\phi}\left(\boldsymbol{\omega}^{\prime}\right) p_{\infty}\left(\boldsymbol{\omega} \cdot \boldsymbol{\omega}^{\prime}\right) d \boldsymbol{\omega}^{\prime}
$$

which imposes that $\zeta(\boldsymbol{r}, \boldsymbol{\omega}) \hat{\phi}(\boldsymbol{\omega})$ is isotropic (because of the isotropy of $\left.p_{\infty}\right)$. Introducing the notation $k(\boldsymbol{r})=\zeta(\boldsymbol{r}, \boldsymbol{\omega}) \hat{\phi}(\boldsymbol{\omega})$, the expression of $\tilde{v}$ becomes that of Eq. (2) with only $K(\boldsymbol{r})$ replaced by $K(\boldsymbol{r})+k(\boldsymbol{r})$. All $v$ solutions have therefore the same form with only $K(\boldsymbol{r})$ taking different values. We checked numerically that the direction-change frequency model of Eq. (2) indeed tends to create stationary distributions such that $\hat{\phi}$ and $\hat{\boldsymbol{\alpha}}$ are close to $\phi$ and $\boldsymbol{\alpha}$ (for large domains and null-flux boundary conditions).

The second inversion property permits addressing some cases in which the previous conditions are not fulfilled, i.e., when the transport equivalence $(\mathrm{Kn} \ll 1)$ cannot be used and the phase function $p_{\infty}$ is anisotropic: If $f_{\text {stat }}$ is observed to be isotropic in the vicinity of a given location $\boldsymbol{r}$ (as in the example of Fig. 1), no other conditions are required, and the directionchange frequency of our Boltzmann's walkers at $\boldsymbol{r}$ is again given by Eq. (2) with $\phi=\hat{\phi}$ and $\boldsymbol{\alpha}=\hat{\boldsymbol{\alpha}}$, but now $\hat{\phi}$ is constant ( $\frac{1}{2 \pi}$ in two dimensions and $\frac{1}{4 \pi}$ in three dimensions). $K(\boldsymbol{r})$ is again a free scalar field. The proof is identical to the preceding one [51]. This property tells us that when the distribution of the walkers is observed to be isotropic at the stationary state, then the angular dependency of the direction-change frequency can be fully obtained by measuring nothing more than the relative gradient of the local density of walkers, i.e., a quantity that is most commonly accessible via simple video analysis and does not require any path-tracking procedure (see Fig. 1).

\section{DISCUSSION}

We expect the direction-change model proposed in this paper to be used for theoretical studies of boundary following because stationary spatial and angular distributions as well as residence times can be exactly calculated, e.g., permitting investigating the effect of the geometry of the boundary [52]. Moreover, in the perspective of experimental studies, the presented inversion properties permit directly formulating and parametrizing a spatially resolved boundary-following model on the basis of easily measurable fields. In many cases, this model containing the free parameter $K$ may be already satisfying, and the fact that the macroscopic fields do not depend on $K$ (which is the direction-averaged value of the direction-change frequency) might even be considered as an invariance property leading to interesting biological interpretations. Otherwise, if a unique model (i.e., without free parameters) is required, more detailed experimental data, such as trajectories, will be needed.

\section{APPENDIX}

The residence-time statistics of diffusion random walks have been shown to satisfy invariance properties $[48,49]$ that have then been gradually enriched and applied to wider classes of random walkers along the following line [50,53-58]: In any stationary state within a given domain $\mathcal{D}$ of dimension $d$, the average time $\langle T\rangle$ spent by constant-speed walkers within a subdomain $\Omega$, from their entry through $\Omega$ 's boundary, noted $\partial \Omega$, to their first return to $\partial \Omega$, equals the ratio of the number $N_{\Omega \text {, stat }}$ of walkers within $\Omega$ to the incoming flux $\hat{\phi}_{\partial \Omega \text {, stat }}$ at $\partial \Omega$,

$$
\langle T\rangle=\frac{N_{\Omega, \text { stat }}}{\hat{\phi}_{\partial \Omega, \text { stat }}}=\frac{\int_{\Omega} d \boldsymbol{r} \int_{\mathcal{S}} d \boldsymbol{\omega} f_{\text {stat }}}{\int_{\partial \Omega} d \boldsymbol{r} \int_{\mathcal{S}_{\text {in }}} d \boldsymbol{\omega} f_{\text {stat }} c \boldsymbol{\omega} \cdot \boldsymbol{n}},
$$

where $\mathcal{S}$ is the $d$-dimensional unit sphere, $\boldsymbol{n}$ is the inward normal to $\partial \Omega, \mathcal{S}_{\text {in }}$ is the corresponding inward hemisphere, $f_{\text {stat }} \equiv f_{\text {stat }}(\boldsymbol{r}, \boldsymbol{\omega}, t)$ is the distribution function of location $\boldsymbol{r}$ and movement direction $\omega$ at time $t$, and $c$ is the movement speed (considered to be constant in this paper). Therefore, $\langle T\rangle$ is invariant to any modification of the characteristics of the walk that leaves $N_{\Omega \text {,stat }}$ and $\hat{\phi}_{\partial \Omega \text {, stat }}$ unchanged. This is in particular the case for the wide class of walkers that lead to an isotropic homogeneous equilibrium. Replacing $f_{\text {stat }}$ by a homogeneous isotropic field in Eq. (A1) shows that such walkers have in common that $\langle T\rangle_{\mathrm{ref}}^{2 d}=\frac{1}{c} \frac{\pi S}{P}$ for $d=2$ (where $S$ is the surface of $\Omega$ and $P$ is the length of $\partial \Omega)$ and $\langle T\rangle_{\text {ref }}^{3 d}=\frac{1}{c} \frac{4 V}{S}$ for $d=3$ (where $V$ is the volume of $\Omega$ and $S$ is the surface of $\partial \Omega$ ). The main point is that $\langle T\rangle_{\text {ref }}$ is invariant to $v$ and $p$ : The stationary average residence-path length $c\langle T\rangle$ only depends on the geometry of $\Omega$.
[1] R. S. Schick, S. R. Loarie, F. Colchero, B. D. Best, A. Boustany, D. A. Conde, P. N. Halpin, L. N. Joppa, C. M. McClellan, and J. S. Clark, Ecol. Lett. 11, 1338 (2008).
[2] R. Nathan, W. M. Getz, E. Revilla, M. Holyoak, R. Kadmon, D. Saltz, and P. E. Smouse, Proc. Natl. Acad. Sci. USA 105, 19052 (2008). 
[3] E. Codling, M. Plank, and S. Benhamou, J. R. Soc., Interface 5 , 813 (2008).

[4] P. Fauchald and T. Tveraa, Oecologia 149, 383 (2006).

[5] T. Mueller and W. F. Fagan, Oikos 117, 654 (2008).

[6] J. Clobert, J. F. Le Galliard, J. Cote, S. Meylan, and M. Massot, Ecol. Lett. 12, 197 (2009).

[7] J. M. Fryxell, M. Hazell, L. Borger, B. D. Dalziel, D. T. Haydon, J. M. Morales, T. McIntosh, and R. C. Rosatte, Proc. Natl. Acad. Sci. USA 105, 19114 (2008).

[8] E. Gurarie, D. A. Andrews, and K. L. Laidre, Ecol. Lett. 12, 395 (2009).

[9] L. Borger, B. D. Dalziel, and J. M. Fryxell, Ecol. Lett. 11, 637 (2008).

[10] R. Jeanson, S. Blanco, R. Fournier, J. Deneubourg, V. Fourcassié, and G. Theraulaz, J. Theor. Biol. 225, 443 (2003).

[11] E. Casellas, J. Gautrais, R. Fournier, S. Blanco, M. Combe, V. Fourcassie, G. Theraulaz, and C. Jost, J. Theor. Biol. 250, 424 (2008).

[12] E. Codling, N. Hill, J. Pitchford, and S. Simpson, Mar. Ecol.: Prog. Ser. 279, 215 (2004).

[13] V. Calenbuhr, L. Chretien, J. Deneubourg, and C. Detrain, J. Theor. Biol. 158, 395 (1992).

[14] K. Painter, J. Math. Biol. 58, 511 (2009).

[15] M. R. D’Orsogna, M. A. Suchard, and T. Chou, Phys. Rev. E 68, 021925 (2003).

[16] M. Moussaï, E. G. Guillot, M. Moreau, J. Fehrenbach, O. Chabiron, S. Lemercier, J. Pettré, C. Appert-Rolland, P. Degond, and G. Theraulaz, PLoS Comput. Biol. 8, e1002442 (2012).

[17] P. Galajda, J. Keymer, P. Chaikin, and R. Austin, J. Bacteriol. 189, 8704 (2007).

[18] G. S. Fraenkel and D. L. Gunn, The Orientation of Animals: Kineses, Taxes and Compass Reactions (Dover, Oxford, 1961).

[19] R. Campan, in Orientation and Communication in Arthropods, edited by M. Lehrer (Birkhäuser Verlag, Basel, 1997), pp. $1-40$.

[20] T. J. Valone, Oikos 56, 357 (1989).

[21] J. M. Davis and J. A. Stamps, Trends Ecol. Evol. 19, 411 (2004).

[22] M. Challet, V. Fourcassie, S. Blanco, R. Fournier, G. Theraulaz, and C. Jost, Naturwissenschaften 92, 367 (2005).

[23] S. Weitz, S. Blanco, R. Fournier, J. Gautrais, C. Jost, and G. Theraulaz, PLoS One 7, e38588 (2012).

[24] D. J. Levey, B. M. Bolker, J. J. Tewksbury, S. Sargent, and N. M. Haddad, Science 309, 146 (2005).

[25] K. L. Laidre, M. P. Heide-Jørgensen, M. L. Logdson, R. C. Hobbs, P. Heagerty, R. Dietz, O. A. Jørgensen, and M. A. Treble, Mar. Biol. 145, 821 (2004).

[26] E. Naylor, Mar. Freshwater Behav. Physiol. 39, 13 (2006).

[27] M. A. McManus and C. B. Woodson, J. Exp. Biol. 215, 1008 (2012).

[28] D. R. Brillinger, H. K. Preisler, A. A. Ager, J. G. Kie, and B. S. Stewart, Bull. Brazilian Math. Soc. 33, 385 (2002).

[29] Y. Ropert-Coudert and R. P. Wilson, Front. Ecol. Environ. 3, 437 (2005)

[30] C. Rutz and G. C. Hays, Biol. Lett. 5, 289 (2009).

[31] K. Evans, M. Lea, and T. Patterson, Deep Sea Res., Part II 88-89, 1 (2013).

[32] H. H. Wensink and H. Löwen, Phys. Rev. E 78, 031409 (2008).

[33] P. K. Radtke and L. Schimansky-Geier, Phys. Rev. E 85, 051110 (2012).

[34] J. Tailleur and M. Cates, Europhys. Lett. 86, 60002 (2009).
[35] M. B. Wan, C. J. Olson Reichhardt, Z. Nussinov, and C. Reichhardt, Phys. Rev. Lett. 101, 018102 (2008).

[36] O. Chepizhko, E. G. Altmann, and F. Peruani, Phys. Rev. Lett. 110, 238101 (2013).

[37] O. Chepizhko and F. Peruani, Phys. Rev. Lett. 111, 160604 (2013).

[38] S. Charifa and M. Bikdash, IEEE Workshop on Robotic Intelligence in Informationally Structured Space, Nashville, TN, 2009. RIISS'09. (IEEE, Piscataway, NJ, 2009), pp. 38-45.

[39] R. Erban and H. Othmer, SIAM J. Appl. Math. 65, 361 (2004).

[40] R. Erban and H. Othmer, J. Math. Biol. 54, 847 (2007).

[41] P. Fisher, R. Merkl, and G. Gerisch, J. Cell Biol. 108, 973 (1989).

[42] E. L. Ionides, K. S. Fang, R. R. Isseroff, and G. F. Oster, J. Math. Biol. 48, 23 (2004).

[43] A. Chakraborty, M. Singh, D. Lucy, and P. Ridland, Math. Comput. Model. 46, 482 (2007).

[44] A. Khuong, V. Lecheval, R. Fournier, S. Blanco, S. Weitz, J.-J. Bezian, and J. Gautrais, PLoS ONE 8, e76531 (2013).

[45] W. Alt, J. Math. Biol. 9, 147 (1980).

[46] H. G. Othmer, S. R. Dunbar, and W. Alt, J. Math. Biol. 26, 263 (1988).

[47] P. Turchin, Quantitative Analysis of Movement: Measuring and Modeling Population Redistribution in Animals and Plants (Sinauer Associates, Sunderland, MA, 1998), Vol. 1.

[48] S. Blanco and R. Fournier, Phys. Rev. Lett. 97, 230604 (2006).

[49] S. Blanco and R. Fournier, Europhys. Lett. 61, 168 (2003).

[50] A. Mazzolo, J. Phys. A: Math. Theor. 42, 105002 (2009).

[51] Because $p_{\infty}$ is allowed to be anisotropic for the second inversion property, the proof that $\zeta(\boldsymbol{r}, \boldsymbol{\omega}) \hat{\phi}$ is isotropic is slightly different: It can be easily checked that any isotropic function $\zeta(\boldsymbol{r}, \boldsymbol{\omega}) \hat{\phi}$ satisfies Eq. (3). Reciprocally, as $p_{\infty}\left(\boldsymbol{\omega} \cdot \boldsymbol{\omega}^{\prime}\right)$ is a normalized probability density function of $\omega$ and symmetric in $\omega$ and $\boldsymbol{\omega}^{\prime}$, Eq. (3) can be rewritten as $\int_{\mathcal{S}}\left[\zeta\left(\boldsymbol{r}, \boldsymbol{\omega}^{\prime}\right) \hat{\phi}-\zeta(\boldsymbol{r}, \boldsymbol{\omega}) \hat{\phi}\right] p_{\infty}(\boldsymbol{\omega}$. $\left.\boldsymbol{\omega}^{\prime}\right) d \boldsymbol{\omega}^{\prime}=0$. Assuming that $p_{\infty}\left(\boldsymbol{\omega} \cdot \boldsymbol{\omega}^{\prime}\right)$ is strictly positive, the validity of this equation for all $\omega$ [in particular also for $\omega=\omega_{m}$ minimizing $\zeta(\boldsymbol{r}, \boldsymbol{\omega}) \hat{\phi}]$ requires that $\zeta(\boldsymbol{r}, \boldsymbol{\omega}) \hat{\phi}$ be an isotropic function.

[52] The proposed model leads to an emerging curvature sensitivity of the residence times that can be calculated using Eq. (A1) (in the Appendix). For example, the average residence time in a band of width $d$ around a circular boundary of radius $R$, assuming $\hat{\boldsymbol{\alpha}}(\boldsymbol{r})=-\alpha \boldsymbol{e}_{\boldsymbol{r}}$ to be a constant-value vector pointing toward the boundary and $\phi=\frac{1}{2 \pi}$ to be constant in Eq. (2), is $\frac{\pi\left(R+\frac{1}{\alpha}\right)\left(e^{\alpha d}-1\right)-d}{c \alpha(R+d)}$, which is an increasing function of $R$. Note that there is no curvature dependency in the model itself.

[53] O. Bénichou, M. Coppey, M. Moreau, P. Suet, and R. Voituriez, Europhys. Lett. 70, 42 (2005).

[54] S. Condamin, O. Bénichou, and M. Moreau, Phys. Rev. E 75, 021111 (2007).

[55] D. S. Grebenkov, Phys. Rev. E 76, 041139 (2007).

[56] A. Zoia, E. Dumonteil, and A. Mazzolo, Europhys. Lett. 100, 40002 (2012).

[57] A. Zoia, E. Dumonteil, and A. Mazzolo, Phys. Rev. E 84, 021139 (2011).

[58] S. Redner, A Guide to First-Passage Processes (Cambridge University Press, Cambridge, UK, 2001).

[59] C. Jost, J. Verret, E. Casellas, J. Gautrais, M. Challet, J. Lluc, S. Blanco, M. Clifton, and G. Theraulaz, J. R. Soc., Interface 4, 107 (2007). 